\title{
Common $\alpha$-Fuzzy Fixed Point Results with Applications to Volterra Integral Inclusions
}

\begin{abstract}
Abdullah Eqal Al-Mazrooei and Jamshaid Ahmad
Department of Mathematics, University of Jeddah, P.O.Box 80327, Jeddah 21589, Saudi Arabia

Correspondence should be addressed to Jamshaid Ahmad; jamshaid_jasim@yahoo.com

Received 16 March 2021; Accepted 28 April 2021; Published 12 May 2021

Academic Editor: Ljubisa Kocinac

Copyright (C) 2021 Abdullah Eqal Al-Mazrooei and Jamshaid Ahmad. This is an open access article distributed under the Creative Commons Attribution License, which permits unrestricted use, distribution, and reproduction in any medium, provided the original work is properly cited.

The purpose of this paper is to establish some common $\alpha$-fuzzy fixed point theorems for a pair fuzzy mappings and obtain some results of literature for multivalued mappings. For it, we define the notion of generalized $\Theta$-contractions in the context of $b$-metric spaces. As applications, we investigate the solutions of Volterra integral inclusions by our established results.
\end{abstract}

\section{Introduction and Preliminaries}

Among all the impressive and inspiring generalizations of metric spaces, $b$-metric space has an integral place. Czerwik [1] in 1993 extended the notion of metric space by introducing the conception of $b$-metric space in this way.

Definition 1. Let $\mathscr{M} \neq \varnothing$. A mapping $d_{b}: \mathscr{M} \times \mathscr{M} \longrightarrow \mathbb{R}_{0}^{+}$is called $b$-metric if it satisfies these assertions:

$$
\begin{aligned}
& \left(b_{1}\right) d_{b}(\omega, \omega)=0 \Leftrightarrow \omega=\omega \\
& \left(b_{2}\right) d_{b}(\omega, \omega)=d_{b}(\omega, \omega) \\
& \left(b_{3}\right) d_{b}(\omega, v) \leq s\left(d_{b}(\omega, \omega)+d_{b}(\Phi, v)\right)
\end{aligned}
$$

for all $\omega, \oplus, v \in \mathscr{M}$, where $s \geq 1$.

Then, $\left(\mathscr{M}, d_{b}, s\right)$ is called a $b$-metric space. A standard example of $b$-metric space which is not metric space is the following:

$\mathscr{M}=\mathbb{R}$ and $d_{b}: \mathscr{M} \times \mathscr{M} \longrightarrow \mathbb{R}$ defined by

$$
d_{b}(\omega, \oplus)=|\omega-\Phi|^{2}
$$

for all $\omega, \oplus \in \mathscr{M}$ with $s=2$.

Let $P_{c b}(\mathscr{M})$ represent the class of all nonempty, bounded, and closed subsets of $\mathscr{M}$. For $\Xi_{1}, \Xi_{2}, \Xi_{3} \in P_{c b}(\mathscr{M})$, we define $H_{b}: P_{c b}(\mathscr{M}) \times P_{c b}(\mathscr{M}) \longrightarrow \mathbb{R}^{+}$by

$$
H_{b}\left(\Xi_{1}, \Xi_{2}\right)=\max \left\{\delta_{b}\left(\Xi_{1}, \Xi_{2}\right), \delta_{b}\left(\Xi_{2}, \Xi_{1}\right),\right\}
$$

where

$$
\begin{aligned}
& \delta_{b}\left(\Xi_{1}, \Xi_{2}\right)=\sup \left\{d_{b}(\omega, \omega): \omega \in \Xi_{1}, \omega \in \Xi_{2}\right\}, \\
& D_{b}\left(\omega, \Xi_{3}\right)=D_{b}\left(\{\omega\}, \Xi_{3}\right)=\inf \left\{d_{b}(\omega, v): \omega \in \Xi_{1}, v \in \Xi_{3}\right\} .
\end{aligned}
$$

Note that $H_{b}$ is called the Hausdorff $b$-metric induced by the $b$-metric $d_{b}$. We recall the following properties from [1-3].

Lemma 1 (see [2]). Let $\left(\mathscr{M}, d_{b}, s\right)$ be a b-metric space. For any $\Xi_{1}, \Xi_{2}, \Xi_{3} \in P_{c b}(\mathscr{M})$ and any $\omega, \omega \in \mathscr{M}$, we have the following:

(i) $D_{b}\left(\omega, \Xi_{2}\right) \leq d_{b}(\omega, b)$ for any $b \in \Xi_{2}$

(ii) $\delta_{b}\left(\Xi_{1}, \Xi_{2}\right) \leq H_{b}\left(\Xi_{1}, \Xi_{2}\right)$

(iii) $D_{b}\left(\omega, \Xi_{2}\right) \leq H_{b}\left(\Xi_{1}, \Xi_{2}\right)$ for any $\omega \in \Xi_{1}$

(iv) $H_{b}\left(\Xi_{1}, \Xi_{2}\right)=0$

(v) $H_{b}\left(\Xi_{1}, \Xi_{2}\right)=H_{b}\left(\Xi_{2}, \Xi_{1}\right)$

(vi) $H_{b}\left(\Xi_{1}, \Xi_{3}\right) \leq s\left[H_{b}\left(\Xi_{1}, \Xi_{2}\right)+H_{b}\left(\Xi_{2}, \Xi_{3}\right)\right]$

(vii) $D_{b}\left(\omega, \Xi_{1}\right) \leq s\left[d_{b}(\omega, \oplus)+d_{b}\left(\omega, \Xi_{1}\right)\right]$.

Later on, many authors (see [4-7]) worked in this way. Recently Jleli and Samet [8] gave the notion of $\Theta$-contractions and proved a contemporary result for such contractions in generalized metric spaces. Afterwards, Hancer 
et al. [9] revised the foregoing definitions by including a broad condition $\left(\Theta_{4}\right)$. Inspired by Jleli and Samet [8] and Hancer et al. [9], Alamri et al. [10] initiated the above notions in the context of $b$-metric spaces and introduced a more general condition $\left(\Theta_{5}\right)$ along with above axioms.

Definition 2 (see [10]). We represent by $\Omega_{s}(s \geq 1)$ the family of all mappings $\Theta: \mathbb{R}^{+} \longrightarrow(1, \infty)$ satisfying these properties:

$\left(\Theta_{1}\right) 0<\varrho_{1}<\varrho_{2} \longrightarrow \Theta\left(\varrho_{1}\right) \leq \Theta\left(\varrho_{2}\right)$

$\left(\Theta_{2}\right)$ for $\left\{\varrho_{n}\right\} \subseteq \mathbb{R}^{+}, \lim _{n \longrightarrow \infty} \Theta\left(\varrho_{n}\right)=1$ if and only if $\lim _{n \rightarrow \infty}\left(\varrho_{n}\right)=0$

$\left(\Theta_{3}\right)$ there exists $h \in(0,1)$ and $\mathfrak{q} \in(0, \infty]$ such that $\lim _{\varrho \longrightarrow 0^{+}}\left(\Theta(\varrho)-1 / \varrho^{h}\right)=\mathfrak{q}$

$\left(\Theta_{4}\right) \Theta(\inf \Xi)=\inf \Theta(\Xi)$ for all $\Xi \subset(0, \infty)$ with $\inf \Xi>0$

$\left(\Theta_{5}\right)$ for all $\left\{\varrho_{n}\right\} \subseteq \mathbb{R}^{+}$such that $\Theta\left(s \varrho_{n}\right) \leq\left[\Theta\left(\varrho_{n-1}\right)\right]^{k}$, $\forall n \in \mathbb{N}$ and some $k \in(0,1)$, then $\Theta\left(s_{n}^{n} \mathrm{\varrho}\right) \leq\left[\Theta\left(s^{n-1} \varrho_{n-1}\right)\right]^{k}$, for all $n \in \mathbb{N}$

They supported this condition by the following nontrivial example.

Example 1 (see [10]). Let $\Theta:(0, \infty) \longrightarrow(1, \infty)$ be given by $\theta(\eta)=e^{\sqrt{\eta e^{\eta}}}$. Clearly, $\Theta$ satisfies $\left(\Theta_{1}\right)-\left(\Theta_{5}\right)$. Here we show only $\left(\Theta_{5}\right)$. Assume that, for all $n \in \mathbb{N}$ and some $k \in(0,1)$, we have $\theta\left(s \varrho_{n}\right) \leq\left[\theta\left(\varrho_{n-1}\right)\right]^{k}$, which implies that

$$
\begin{aligned}
& e^{\sqrt{s \varrho_{n} e^{s \varrho_{n}}}} \leq\left[e^{\sqrt{\varrho_{n-1} e^{\varrho_{n-1}}}}\right]^{k}, \\
& \sqrt{s \varrho_{n} e^{s \varrho_{n}}} \leq k \sqrt{\varrho_{n-1} e^{\varrho_{n-1}}} .
\end{aligned}
$$

This implies that

$$
\sqrt{s \varrho_{n} e^{s \varrho_{n}-\varrho_{n-1}}} \leq k \sqrt{\varrho_{n-1}}
$$

As $\theta\left(s \varrho_{n}\right) \leq\left[\theta\left(\varrho_{n-1}\right)\right]^{k} \leq \theta\left(\varrho_{n-1}\right)$. Also $\theta$ is nondecreasing, so $s \varrho_{n} \leq \varrho_{n-1}$ and $s \varrho_{n}-\varrho_{n-1} \leq 0$ implies $e^{s^{n-1}\left(s \varrho_{n}-\varrho_{n-1}\right)} \leq e^{s \varrho_{n}-\varrho_{n-1}}$. Therefore, (5) implies

$$
\begin{gathered}
\sqrt{s \varrho_{n} e^{s^{n-1}}\left(s \varrho_{n}-\varrho_{n-1}\right)} \leq k \sqrt{\varrho_{n-1}} \Rightarrow \sqrt{\frac{s \varrho_{n} e^{s^{n} \varrho_{n}}}{e^{s^{n-1} \varrho_{n-1}}}} \leq k \sqrt{\varrho_{n-1}} \\
\Rightarrow \sqrt{s \varrho_{n} e^{s^{n} \varrho_{n}}} \leq k \sqrt{\varrho_{n-1} e^{s^{n-1} \varrho_{n-1}}} \Rightarrow \sqrt{s^{n} \varrho_{n} e^{s^{n} \varrho_{n}}} \leq k \sqrt{s^{n-1} \varrho_{n-1} e^{s^{n-1} \varrho_{n-1}}} \\
\Rightarrow e^{\sqrt{s^{n} \varrho_{n} e^{s^{n} \varrho_{n}}}} \leq e^{k \sqrt{s^{n-1} \varrho_{n-1} e^{s^{n-1} \varrho_{n-1}}}} \Rightarrow \theta\left(s^{n} \varrho_{n}\right) \leq\left[\theta\left(s^{n-1} \varrho_{n-1}\right)\right]^{k},
\end{gathered}
$$

and hence $\left(\Theta_{5}\right)$ holds.

On the other side, Kumam et al. [11] utilized the concept of $b$-metric space and obtained common $\alpha$ - fuzzy fixed points for fuzzy mappings under generalized rational contractions. For more details in the direction of fixed point results for fuzzy mappings, we refer [9-20] to the readers.

We need the following lemma of Czerwik [2].

Lemma 2 (see [11]). Let $\left(\mathscr{M}, d_{b}, s\right)$ be a b-metric space and $\Xi_{1}, \Xi_{2} \in C B(\mathscr{M})$, then $\forall \omega \in \Xi_{1}$,

$$
d_{b}\left(\omega, \Xi_{2}\right) \leq H_{b}\left(\Xi_{1}, \Xi_{2}\right) \text {. }
$$

In this paper, we obtain common $\alpha$-fuzzy fixed point results for a pair of fuzzy mappings and establish some theorems to generalize some results from the literature. We solve the Volterra integral inclusions as application of our established results.

\section{Main Results}

In this way, we state our main result.

Theorem 1. Let $\left(\mathscr{M}, d_{b}, s\right)$ be a complete b-metric space with coefficient $s \geq 1$ such that $d_{b}$ is continuous. Assume that $\mathcal{O}_{1}, \mathcal{O}_{2}: \mathscr{M} \longrightarrow \mathscr{F}(\mathscr{M})$ and for each $\omega, \omega \in \mathscr{M}$, there exist $\alpha_{\mathscr{O}_{1}}(\omega), \alpha_{\mathscr{O}_{2}}(\Phi) \in(0,1] \quad$ such that $\left[\mathscr{O}_{1} \omega\right]_{\alpha_{\mathscr{O}_{1}}(\omega)},\left[\mathcal{O}_{2} \oplus\right]_{\alpha_{\mathscr{O}_{2}}(\varpi)} \in P_{c b}(\mathscr{M})$. If there exist $\Theta \in \Omega_{s}$ and $k \in(0,1)$ such that

$$
\Theta\left(s H_{b}\left(\left[\mathcal{O}_{1} \omega\right]_{\alpha_{\mathscr{O}_{1}}(\omega)},\left[\mathcal{O}_{2} \omega\right]_{\alpha_{\mathscr{O}_{2}}(\varpi)}\right)\right) \leq\left[\Theta\left(d_{b}(\omega, \omega)\right)\right]^{k},
$$

for all $\omega, \omega \in \mathscr{M}$ with $H_{b}\left(\left[\mathcal{O}_{1} \omega\right]_{\alpha_{\mathscr{O}_{1}}(\omega)},\left[\mathcal{O}_{2} \omega\right]_{\alpha_{\mathscr{O}_{2}}(\varpi)}\right)>0$, then there exists $\omega^{*} \in \mathscr{M}$ such that $\omega^{*} \in\left[\mathscr{O}_{1} \omega^{*}\right]_{\alpha_{\mathscr{O}_{1}}\left(\omega^{*}\right)} \cap$ $\left[\mathcal{O}_{2} \omega^{*}\right]_{\alpha_{\mathcal{O}_{2}}\left(\omega^{*}\right)}$.

Proof.

Let $\omega_{0} \in \mathscr{M}$, then by assumption there exists $\alpha_{\mathscr{O}_{1}}\left(\omega_{0}\right) \in(0,1]$ such that $\left[\mathscr{O}_{1} \omega_{0}\right]_{\alpha_{\mathscr{O}_{1}}\left(\omega_{0}\right)} \in C B(\mathscr{M})$. Let $\omega_{1} \in\left[\mathcal{O}_{1} \omega_{0}\right]_{\alpha_{\mathcal{O}_{1}}\left(\omega_{0}\right)}$. For this $\omega_{1}$, there exists $\alpha_{\mathcal{O}_{2}}\left(\omega_{1}\right) \in(0,1]$ such that $\left[\mathcal{O}_{2} \omega_{1}\right]_{\alpha_{\mathcal{O}_{2}}\left(\omega_{1}\right)} \in P_{c b}(\mathscr{M})$. By Lemma $2,\left(\Theta_{1}\right)$ and (8), we have

$$
\Theta\left(s d\left(\omega_{1},\left[\mathcal{O}_{2} \omega_{1}\right]_{\alpha_{\mathscr{O}_{2}}\left(\omega_{1}\right)}\right)\right) \leq \Theta\left(s H_{b}\left(\left[\mathcal{O}_{1} \omega_{0}\right]_{\alpha_{\mathscr{O}_{1}}\left(\omega_{0}\right)},\left[\mathcal{O}_{2} \omega_{1}\right]_{\alpha_{\mathscr{O}_{2}}}\left(\omega_{1}\right)\right)\right) \leq\left[\Theta\left(d_{b}\left(\omega_{0}, \omega_{1}\right)\right)\right]^{k} .
$$


Thus,

$$
\Theta\left(s d\left(\omega_{1},\left[\mathcal{O}_{2} \omega_{1}\right]_{\alpha_{\sigma_{2}}\left(\omega_{1}\right)}\right)\right) \leq\left[\Theta\left(d_{b}\left(\omega_{0}, \omega_{1}\right)\right)\right]^{k} .
$$

From $\left(\Theta_{4}\right)$, we know that

$$
\Theta\left(s d\left(\omega_{1},\left[\mathcal{O}_{2} \omega_{1}\right]_{\alpha_{\mathscr{O}_{2}}\left(\omega_{1}\right)}\right)\right)=\inf _{y \in\left[\mathcal{O}_{2} \omega_{1}\right]_{\alpha_{\mathcal{O}_{2}}\left(\omega_{1}\right)}} \Theta\left(s d_{b}\left(\omega_{1}, y\right)\right) .
$$

Thus from (10), we get

$$
\inf _{y \in\left[\mathcal{O}_{2} \omega_{1}\right]_{\alpha_{\mathcal{O}_{2}}\left(\omega_{1}\right)}} \Theta\left(s d_{b}\left(\omega_{1}, y\right)\right) \leq\left[\Theta\left(d_{b}\left(\omega_{0}, \omega_{1}\right)\right)\right]^{k}
$$

Then, from (12), there exists $\omega_{2} \in\left[\mathcal{O}_{2} \omega_{1}\right]_{\alpha_{\mathscr{O}_{2}}\left(\omega_{1}\right)}$ (obviously, $\left.\omega_{2} \neq \omega_{1}\right)$ such that

$$
\Theta\left(s d_{b}\left(\omega_{1}, \omega_{2}\right)\right) \leq\left[\Theta\left(d_{b}\left(\omega_{0}, \omega_{1}\right)\right)\right]^{k} .
$$

For this $\omega_{2}$, there exists $\alpha_{\mathscr{O}_{1}}\left(\omega_{2}\right) \in(0,1]$ such that $\left[\mathcal{O}_{1} \omega_{2}\right]_{\alpha_{\mathcal{O}_{1}}\left(\omega_{2}\right)} \in P_{c b}(\mathscr{M})$. By Lemma $2,\left(\Theta_{1}\right)$, and (8), we have

$$
\Theta\left(s d_{b}\left(\omega_{2},\left[\mathcal{O}_{1} \omega_{2}\right]_{\alpha_{\sigma_{1}}\left(\omega_{2}\right)}\right)\right) \leq \Theta\left(s H_{b}\left(\left[\mathcal{O}_{2} \omega_{1}\right]_{\alpha_{\mathscr{O}_{2}}\left(\omega_{1}\right)},\left[\mathcal{O}_{1} \omega_{2}\right]_{\alpha_{\mathscr{O}_{1}}\left(\omega_{2}\right)}\right)\right) \leq\left[\Theta\left(d_{b}\left(\omega_{1}, \omega_{2}\right)\right)\right]^{k} .
$$

Thus,

$\Theta\left(s d_{b}\left(\omega_{2},\left[\mathcal{O}_{1} \omega_{2}\right]_{\alpha_{\mathscr{O}_{1}}}\left(\omega_{2}\right)\right)\right) \leq\left[\Theta\left(d_{b}\left(\omega_{1}, \omega_{2}\right)\right)\right]^{k}$

From $\left(\Theta_{4}\right)$, we know that

$\Theta\left(s d_{b}\left(\omega_{2},\left[\mathcal{O}_{1} \omega_{2}\right]_{\alpha_{\mathscr{O}_{1}}\left(\omega_{2}\right)}\right)\right)=\inf _{y \in\left[\mathcal{O}_{1} \omega_{2}\right]_{\alpha_{\mathscr{O}_{1}}\left(\omega_{2}\right)}} \Theta\left(s d_{b}\left(\omega_{2}, y\right)\right)$.

Thus from (15), we get

$$
\inf _{y \in\left[\mathcal{O}_{1} \omega_{2}\right]_{\alpha_{\mathcal{O}_{1}}\left(\omega_{2}\right)}} \Theta\left(s d_{b}\left(\omega_{2}, y\right)\right) \leq\left[\Theta\left(d_{b}\left(\omega_{1}, \omega_{2}\right)\right)\right]^{k} .
$$

Then, from (17), there exists $\omega_{3} \in\left[\mathscr{O}_{1} \omega_{2}\right]_{\alpha_{\mathscr{O}_{1}}\left(\omega_{2}\right)}$ (obviously, $\left.\omega_{3} \neq \omega_{2}\right)$ such that

$$
\Theta\left(s d_{b}\left(\omega_{2}, \omega_{3}\right)\right) \leq\left[\Theta\left(d_{b}\left(\omega_{1}, \omega_{2}\right)\right)\right]^{k} .
$$

So, continuing in the same way, we construct $\left\{\omega_{n}\right\}$ in $\mathscr{M}$ such that

$$
\begin{gathered}
\omega_{2 n+1} \in\left[\mathcal{O}_{1} \omega_{2 n}\right]_{\alpha_{\mathcal{O}_{1}}\left(\omega_{2 n}\right)}, \\
\omega_{2 n+2} \in\left[\mathcal{O}_{2} \omega_{2 n+1}\right]_{\alpha_{\widehat{\sigma}_{2}}\left(\omega_{2 n+1}\right)}, \\
\Theta\left(s d_{b}\left(\omega_{2 n+1}, \omega_{2 n+2}\right)\right) \leq\left[\Theta\left(d_{b}\left(\omega_{2 n}, \omega_{2 n+1}\right)\right)\right]^{k}, \\
\Theta\left(s d_{b}\left(\omega_{2 n+2}, \omega_{2 n+3}\right)\right) \leq\left[\Theta\left(d_{b}\left(\omega_{2 n+1}, \omega_{2 n+2}\right)\right)\right]^{k},
\end{gathered}
$$

for all $n \in \mathbb{N}$. From (20) and (21), we get

$$
\Theta\left(s d_{b}\left(\omega_{n}, \omega_{n+1}\right)\right) \leq\left[\Theta\left(d_{b}\left(\omega_{n-1}, \omega_{n}\right)\right)\right]^{k},
$$

for all $n \in \mathbb{N}$. It follows by $(22)$ and property $\left(\Theta_{5}\right)$ that

$$
\Theta\left(s^{n} d_{b}\left(\omega_{n}, \omega_{n+1}\right)\right) \leq\left[\Theta\left(s^{n-1} d_{b}\left(\omega_{n-1}, \omega_{n}\right)\right)\right]^{k},
$$

which further implies that

$$
\Theta\left(s^{n} d_{b}\left(\omega_{n}, \omega_{n+1}\right)\right) \leq\left[\Theta\left(s^{n-1} d_{b}\left(\omega_{n-1}, \omega_{n}\right)\right)\right]^{k} \leq\left[\Theta\left(s^{n-2} d_{b}\left(\omega_{n-2}, \omega_{n-1}\right)\right)\right]^{k^{2}} \leq \cdots \leq\left[\Theta\left(d_{b}\left(\omega_{0}, \omega_{1}\right)\right)\right]^{k^{n}}
$$

$$
\left|\frac{\Theta\left(s^{n} d_{b}\left(\omega_{n}, \omega_{n+1}\right)\right)-1}{\left(s^{n} d_{b}\left(\omega_{n}, \omega_{n+1}\right)\right)^{r}}-\mathfrak{q}\right| \leq \varsigma_{2},
$$

$$
\Theta\left(s^{n} d_{b}\left(\omega_{n}, \omega_{n+1}\right)\right) \leq\left[\Theta\left(d_{b}\left(\omega_{0}, \omega_{1}\right)\right)\right]^{k^{n}},
$$

for all $n \in \mathbb{N}$. Since $\Theta \in \Omega$, so letting $n \longrightarrow \infty$ in (25), we get

$$
\lim _{n \longrightarrow \infty} \Theta\left(s^{n} d_{b}\left(\omega_{n}, \omega_{n+1}\right)\right)=1 .
$$

This implies

$$
\lim _{n \longrightarrow \infty} s^{n} d_{b}\left(\omega_{n}, \omega_{n+1}\right)=0,
$$

by $\left(\Theta_{2}\right)$. By $\left(\Theta_{3}\right)$, there exists $0<r<1$ and $\mathfrak{q} \in(0, \infty]$ so that

$$
\lim _{n \rightarrow \infty} \frac{\Theta\left(s^{n} d_{b}\left(\omega_{n}, \omega_{n+1}\right)\right)-1}{\left(s^{n} d_{b}\left(\omega_{n}, \omega_{n+1}\right)\right)^{r}}=\mathfrak{q} .
$$

Suppose that $\mathfrak{q}<\infty$. For this case, let $\varsigma_{2}=(\mathfrak{q} / 2)>0$. By definition of the limit, there exists $n_{0} \in \mathbb{N}$ so that

for all $n>n_{0}$. This implies that

$$
\frac{\Theta\left(s^{n} d_{b}\left(\omega_{n}, \omega_{n+1}\right)\right)-1}{\left(s^{n} d_{b}\left(\omega_{n}, \omega_{n+1}\right)\right)^{r}} \geq \mathfrak{q}-\varsigma_{2}=\frac{\mathfrak{q}}{2}=\varsigma_{2},
$$

for all $n>n_{0}$. Then

$$
n\left(s^{n} d_{b}\left(\omega_{n}, \omega_{n+1}\right)\right)^{r} \leq \varsigma_{1} n\left[\Theta\left(s^{n} d_{b}\left(\omega_{n}, \omega_{n+1}\right)\right)-1\right],
$$

for all $n>n_{0}$, where $\varsigma_{1}=\left(1 / \varsigma_{2}\right)$. Now we assume that $\mathfrak{q}=\infty$. Let $\varsigma_{2}>0$. From the definition of the limit, there exists $n_{0} \in \mathbb{N}$ such that

$$
\varsigma_{2} \leq \frac{\Theta\left(s^{n} d_{b}\left(\omega_{n}, \omega_{n+1}\right)\right)-1}{\left(s^{n} d_{b}\left(\omega_{n}, \omega_{n+1}\right)\right)^{r}},
$$

for all $n>n_{0}$, which implies 


$$
n\left(s^{n} d_{b}\left(\omega_{n}, \omega_{n+1}\right)\right)^{r} \leq \varsigma_{1} n\left[\Theta\left(s^{n} d_{b}\left(\omega_{n}, \omega_{n+1}\right)\right)-1\right],
$$

for all $n>n_{0}$, where $\varsigma_{1}=\left(1 / \varsigma_{2}\right)$. Hence, in all cases, there exists $\varsigma_{1}>0$ and $n_{0} \in \mathbb{N}$ such that

$$
n\left(s^{n} d_{b}\left(\omega_{n}, \omega_{n+1}\right)\right)^{r} \leq \varsigma_{1} n\left[\Theta\left(s^{n} d_{b}\left(\omega_{n}, \omega_{n+1}\right)\right)-1\right],
$$

for all $n>n_{0}$. Hence by (25) and (34), we obtain

$$
n\left(s^{n} d_{b}\left(\omega_{n}, \omega_{n+1}\right)\right)^{r} \leq \varsigma_{1} n\left(\left[\Theta\left(d_{b}\left(\omega_{0}, \omega_{1}\right)\right)\right]^{r^{n}}-1\right) .
$$

Taking the limit $n \longrightarrow \infty$, we get

$$
\lim _{n \longrightarrow \infty} n\left(s^{n} d_{b}\left(\omega_{n}, \omega_{n+1}\right)\right)^{r}=0 .
$$

Thus $\lim _{n \longrightarrow \infty} n^{(1 / r)} s^{n} d_{b}\left(\omega_{n}, \omega_{n+1}\right)=0$ which implies that $\sum_{n=1}^{\infty} s^{n} d_{b}\left(\omega_{n}, \omega_{n+1}\right)$ is convergent. Thus $\left\{\omega_{n}\right\}$ is a Cauchy sequence in $\mathscr{M}$. Since $\left(\mathscr{M}, d_{b}, s\right)$ is a complete $b$-metric space, so there exists a $\omega^{*} \in \mathscr{M}$ such that

$$
\lim _{n \longrightarrow \infty} \omega_{n}=\omega^{*} \text {. }
$$

Now, we prove that $\omega^{*} \in\left[\mathcal{O}_{2} \omega^{*}\right]_{\alpha_{\sigma_{2}}\left(\omega^{*}\right)}$. We suppose on the contrary that $\omega^{*} \notin\left[\mathcal{O}_{2} \omega^{*}\right]_{\alpha_{\sigma_{2}}\left(\omega^{*}\right)}$, then there exist $n_{0} \in \mathbb{N}$ and $\left\{\omega_{n_{k}}\right\}$ of $\left\{\omega_{n}\right\}$ such that $d_{b}\left(\omega_{2 n_{k}+1},\left[\mathcal{O}_{2} \omega^{*}\right]_{\alpha_{\sigma_{2}}\left(\omega^{*}\right)}\right)>0$, for all $n_{k} \geq n_{0}$. Now, using (8) with $\omega=\omega_{2 n_{k}+1}$ and $\omega=\omega^{*}$, we obtain

$$
\begin{aligned}
\Theta\left[d_{b}\left(\omega_{2 n_{k}+1},\left[\mathcal{O}_{2} \omega^{*}\right]_{\alpha_{\mathscr{O}_{2}}\left(\omega^{*}\right)}\right)\right] & \leq \Theta\left[s d_{b}\left(\omega_{2 n_{k}+1},\left[\mathcal{O}_{2} \omega^{*}\right]_{\alpha_{\mathscr{O}_{2}}\left(\omega^{*}\right)}\right)\right] \\
& \leq \Theta\left[s H_{b}\left(\left[\mathcal{O}_{1} \omega_{2 n_{k}}\right]_{\alpha_{\mathscr{O}_{1}}\left(\omega_{2 n_{k}}\right)},\left[\mathcal{O}_{2} \omega^{*}\right]_{\alpha_{\mathscr{O}_{2}}\left(\omega^{*}\right)}\right)\right] \leq\left[\Theta\left(d\left(\omega_{2 n_{k}}, \omega^{*}\right)\right)\right]^{k} .
\end{aligned}
$$

As $k \in(0,1)$, so by $\left(\Theta_{1}\right)$ so we obtain

$$
d_{b}\left(\omega_{2 n_{k}+1},\left[\mathcal{O}_{2} \omega^{*}\right]_{\alpha_{\sigma_{2}}\left(\omega^{*}\right)}\right)<d_{b}\left(\omega_{2 n_{k}}, \omega^{*}\right) .
$$

Letting $n \longrightarrow \infty$, we have

$$
d_{b}\left(\omega^{*},\left[\mathcal{O}_{2} \omega^{*}\right]_{\alpha_{\mathscr{O}_{2}}\left(\omega^{*}\right)}\right) \leq 0 .
$$

Hence, $\omega^{*} \in\left[\mathcal{O}_{2} \omega^{*}\right]_{\alpha_{\sigma_{2}}\left(\omega^{*}\right)}$. Likewise, one can straightforwardly prove that $\omega^{*} \in\left[\mathcal{O}_{1} \omega^{*}\right]_{\alpha_{\mathscr{O}_{1}}\left(\omega^{*}\right)}$. Thus, $\omega^{*} \in\left[\mathcal{O}_{1} \omega^{*}\right]_{\alpha_{\mathscr{O}_{1}}\left(\omega^{*}\right)} \cap\left[\mathcal{O}_{2} \omega^{*}\right]_{\alpha_{\mathscr{O}_{2}}\left(\omega^{*}\right)}$.

Note: From now to onwards, we consider $d_{b}$ as continuous functional and $\left(\mathscr{M}, d_{b}, s\right)$ as complete $b$-metric space.

The following corollary follows from Theorem 1 by considering $\Theta(\eta)=e^{\sqrt{\eta}}$ for $\eta>0$.

Theorem 2. Let $\mathcal{O}_{1}, \mathcal{O}_{2}: \mathscr{M} \longrightarrow \mathscr{F}(\mathscr{M})$, and for each $\omega, \Phi \in \mathscr{M}, \quad \exists \alpha_{\mathscr{O}_{1}}(\omega), \alpha_{\mathscr{O}_{2}}(\Phi) \in(0,1] \quad$ such that $\left[\mathcal{O}_{1} \omega\right]_{\alpha_{\mathcal{O}_{1}}(\omega)},\left[\mathcal{O}_{2} \oplus\right]_{\alpha_{\mathcal{O}_{2}}(\varpi)} \in P_{c b}(\mathscr{M})$. If $\exists k \in(0,1)$ such that

$$
s H_{b}\left(\left[\mathcal{O}_{1} \omega\right]_{\alpha_{\mathscr{O}_{1}}(\omega)},\left[\mathcal{O}_{2} \omega\right]_{\alpha_{\mathscr{O}_{2}}(\varpi)}\right) \leq k d_{b}(\omega, \oplus)
$$

for all $\omega, \oplus \in \mathscr{M}$, then there exists $\omega^{*} \in \mathscr{M}$ such that $\omega^{*} \in\left[\mathcal{O}_{1} \omega^{*}\right]_{\alpha_{\mathscr{O}_{1}}\left(\omega^{*}\right)} \cap\left[\mathcal{O}_{2} \omega^{*}\right]_{\alpha_{\mathscr{O}_{2}}\left(\omega^{*}\right)}$.

Theorem 3. Let $\mathcal{O}: \mathscr{M} \longrightarrow \mathscr{F}(\mathscr{M})$, and for each $\omega, \omega \in \mathscr{M}$, there exist $\alpha_{\mathscr{O}}(\omega), \alpha_{\mathscr{O}}(\Phi) \in(0,1]$ such that $[\mathcal{O} \omega]_{\alpha_{\mathscr{O}}(\omega)},[\mathcal{O} \oplus]_{\alpha_{\mathscr{O}}(\varpi)} \in P_{c b}(\mathscr{M})$. If there exists $k \in(0,1)$ such that

$$
s H_{b}\left([\mathcal{O} \omega]_{\alpha_{\mathscr{O}}(\omega)},[\mathcal{O} \bowtie]_{\alpha_{\mathscr{O}}(\varpi)}\right) \leq k d_{b}(\omega, \oplus),
$$

for all $\omega, \omega \in \mathscr{M}$, then there exists $\omega^{*} \in \mathscr{M}$ such that $\omega^{*} \in\left[\mathcal{O} \omega^{*}\right]_{\alpha_{\mathscr{O}}\left(\omega^{*}\right)}$.

Example 2. Let $\mathscr{M}=\{0,1,2\}$ and $d_{b}: \mathscr{M} \times \mathscr{M} \longrightarrow \mathbb{R}_{0}^{+}$by

$$
d_{b}(\omega, \oplus)= \begin{cases}0, & \text { if } \omega=\omega, \\ \frac{1}{6}, & \text { if } \omega \neq \omega \text { and } \omega, \oplus \in\{0,1\}, \\ \frac{1}{2}, & \text { if } \omega \neq \omega \text { and } \omega, \oplus \in\{0,2\}, \\ 1, & \text { if } \omega \neq \omega \text { and } \omega, \oplus \in\{1,2\} .\end{cases}
$$

It is easy to see that $(\mathscr{M}, d)$ is a complete $b$-metric space with coefficient $s=(3 / 2)$. Define

$$
(\mathscr{O} 0)(\eta)=(\mathscr{O} 1)(\eta)= \begin{cases}\frac{1}{2}, & \text { if } \eta=0, \\ 0, & \text { if } \eta=1,2,\end{cases}
$$

$$
(\mathcal{O} 2)(\eta)= \begin{cases}0, & \text { if } \eta=0,2, \\ \frac{1}{2}, & \text { if } \eta=1 .\end{cases}
$$

Define $\alpha: \mathscr{M} \longrightarrow(0,1]$ by $\alpha(\omega)=(1 / 2)$ for all $\omega \in \mathscr{M}$. Now we obtain that

$$
[\mathcal{O} \omega]_{(1 / 2)}= \begin{cases}\{0\}, & \text { if } \omega=0,1 \\ \{1\}, & \text { if } \omega=2 .\end{cases}
$$




$$
H_{b}\left([\mathcal{O} 1]_{(1 / 2)},[\mathcal{O} 2]_{(1 / 2)}\right)=H_{b}\left([\mathcal{O} 1]_{(1 / 2)},[\mathcal{O} 2]_{(1 / 2)}\right)=H_{b}(\{0\},\{1\})=\frac{1}{6}
$$

Taking $\Theta(\eta)=e^{\sqrt{\eta}}$ for $\eta>0$ and $k=(1 / 2)$. Then

$\Theta\left(s H_{b}\left([\mathcal{O} 0]_{(1 / 2)},[\mathcal{O} 2]_{(1 / 2)}\right)\right)=e^{\left(\frac{1}{4}\right)^{(1 / 2)}}<e^{\left(\frac{1}{2}\right)^{(1 / 4)}}=\left[\Theta\left(d_{b}(0,2)\right)\right]^{k}$,

also

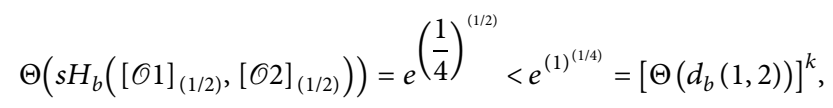

for all $\omega, \oplus \in \mathscr{M}$. As a result, all assertions of Theorem 6 hold and there exists $0 \in \mathscr{M}$ such that $0 \in[00]_{(1 / 2)}$ is an $\alpha$-fuzzy fixed point of $\mathcal{O}$.

\section{Set-Valued Results}

Theorem 4. Let $G_{1}, G_{2}: X \longrightarrow C B(X)$. Suppose that $\exists k \in(0,1)$ such that

$$
\Theta\left(s H_{b}\left(G_{1} \omega, G_{2} \omega\right)\right) \leq\left[\Theta\left(d_{b}(\omega, \omega)\right)\right]^{k},
$$

for all $\omega, \oplus \in \mathscr{M}$. Then there exists $\omega^{*} \in \mathscr{M}$ such that $\omega^{*} \in G_{1} \omega^{*} \cap G_{2} \omega^{*}$.

Proof. Define $\alpha: \mathscr{M} \longrightarrow[0,1]$ and $\mathcal{O}_{1}, \mathcal{O}_{2}: \mathscr{M} \longrightarrow \mathscr{F}(\mathscr{M})$ by

$$
\begin{aligned}
& \mathcal{O}_{1}(\omega)(\eta)= \begin{cases}\alpha(\omega), & \text { if } \eta \in G_{1} \omega, \\
0, & \text { if } \eta \notin G_{1} \omega,\end{cases} \\
& \mathcal{O}_{2}(\omega)(\eta)= \begin{cases}\alpha(\omega), & \text { if } \eta \in G_{2} \omega, \\
0, & \text { if } \eta \notin G_{2} \omega .\end{cases}
\end{aligned}
$$

Then

$$
\begin{aligned}
& {\left[\mathcal{O}_{1} \omega\right]_{\alpha(\omega)}=\left\{\eta: \mathcal{O}_{1}(\omega)(\eta) \geq \alpha(\omega)\right\}=G_{1} \omega,} \\
& {\left[\mathcal{O}_{2} \omega\right]_{\alpha(\omega)}=\left\{\eta: \mathcal{O}_{2}(\omega)(\eta) \geq \alpha(\omega)\right\}=G_{2} \omega .}
\end{aligned}
$$

Thus, Theorem 4 can be applied to get $\omega^{*} \in \mathscr{M}$ such that

$$
\omega^{*} \in\left[\mathcal{O}_{1} \omega^{*}\right]_{\alpha\left(\omega^{*}\right)} \cap\left[\mathcal{O}_{2} \omega^{*}\right]_{\alpha\left(\omega^{*}\right)}=G_{1} \omega^{*} \cap G_{2} \omega^{*} \text {. }
$$

Corollary 1. Let $G: X \longrightarrow C B(X)$ be multivalued mapping. Assume that there exists $k \in(0,1)$ such that

$$
\Theta\left(s H_{b}(G \omega, G \bowtie)\right) \leq\left[\Theta\left(d_{b}(\omega, \varpi)\right)\right]^{k},
$$

for all $\omega, \oplus \in \mathscr{M}$. Then there exists $\omega^{*} \in \mathscr{M}$ such that $\omega^{*} \in G \omega^{*}$.

Remark 1. If $s=1$, then $b$-metric spaces turns into complete metric space and we obtain some new results for fuzzy mappings as well as multivalued mappings in metric spaces.

\section{Applications}

Consider the Volterra integral inclusion

$$
\omega(\kappa) \in \mathfrak{h}(\kappa)+\int_{0}^{\kappa} \mathfrak{J}(\kappa, \tau, \omega(\tau)) \mathrm{d} \tau, \quad \kappa \in[0,1],
$$

where $\mathfrak{\Im}:[0,1] \times[0,1] \times \mathbb{R} \longrightarrow \chi_{c v}(\mathbb{R})$ a given set-valued mapping and $\mathfrak{h}, \omega \in C[0,1]$ be such that $\mathfrak{h}$ is given and $\omega$ is unknown function.

Now, for $p \geq 1$, consider the $b$-metric $d_{b}$ on $C[0,1]$ defined by

$$
d_{b}(\omega, \omega)=\left(\max _{\kappa \in[0,1]}|\omega(\kappa)-\omega(\kappa)|\right)^{p}=\max _{\kappa \in[0,1]}|\omega(\kappa)-\omega(\kappa)|^{p},
$$

for all $\omega, \oplus \in C[0,1]$. Then, $\left(C[0,1], d_{b}, 2^{p-1}\right)$ is a complete $b$-metric space.

We will assume the following:

(a) For each $\omega \in C[0,1]$, the mapping $\mathfrak{J}:[0,1] \times[0,1] \times \mathbb{R} \longrightarrow \chi_{c v}(\mathbb{R})$ is such that $\mathfrak{J}(\kappa, \tau, \omega(\tau))$ is lower semicontinuous in $[0,1] \times[0,1]$

(b) There exists $l:[0,1] \longrightarrow[0,+\infty)$ which is continuous such that

$$
|\mathfrak{\Im}(\kappa, \tau, \omega)-\mathfrak{J}(\kappa, \tau, \oplus)|^{p} \leq l(\tau)|\omega(\tau)-\Phi(\tau)|
$$

for all $\kappa, \tau \in[0,1], \omega, \bowtie \in C[0,1]$.

(c) There exists $k \in(0,1)$ so that

$$
\left(\int_{0}^{\kappa} l(\tau) \mathrm{d} \tau\right)^{p} \leq \frac{k}{2^{p-1}}
$$

Theorem 5. Under the assumptions (a)-(c), the integral inclusion (54) has a solution in $C[0,1]$.

Proof. Let $\mathscr{M}=C[0,1]$. Define $\mathcal{O}: \mathscr{M} \longrightarrow \mathscr{F}(\mathscr{M})$ by

$$
[\mathcal{O} \omega]_{\alpha_{\mathscr{O}}(\omega)}=\left\{\omega \in \mathscr{M}: \Phi(\kappa) \in \mathfrak{h}(\kappa)+\int_{0}^{\kappa} \mathfrak{J}(\kappa, \tau, \omega(\tau)) \mathrm{d} \tau\right\},
$$

for all $\kappa \in[0,1]$. Let $\omega \in \mathscr{M}$ be arbitrary, then there exists $\alpha_{\mathscr{O}}(\omega) \in(0,1]$. For $\mathfrak{\Im}_{\omega}(\kappa, \tau):[0,1] \times[0,1] \longrightarrow \chi_{c v}(\mathbb{R})$, it follows from Michael's selection theorem that there exists $\dot{\mathrm{j}}_{\omega}(\kappa, \tau):[0,1] \times[0,1] \longrightarrow \mathbb{R}$ such that $\dot{\mathfrak{j}}_{\omega}(\kappa, \tau) \in \mathfrak{J}_{\omega}(\kappa, \tau)$ for each $\kappa, \tau \in[0,1]$. It follows that $\mathfrak{h}(\kappa)+\int_{0}^{\kappa} \dot{\mathfrak{t}}_{\omega}$ $(\kappa, \tau) \mathrm{d} \tau \in[\mathcal{O} \omega]_{\alpha_{\odot}(\omega)}$. Hence, $[\mathcal{O} \omega]_{\alpha_{\odot}(\omega)} \neq \varnothing$. It is a simple matter to show that $[\mathcal{O} \omega]_{\alpha_{\mathscr{O}}(\omega)}$ is closed, and so details are 
excluded (see also [17]). Moreover, since $\mathfrak{h}$ is continuous on $[0,1]$, and $\mathfrak{\Im}_{\omega}(\kappa, \tau)$ is continuous, their ranges are bounded. This means that $[\mathcal{O} \omega]_{\alpha_{\mathscr{O}}(\omega)}$ is bounded. Thus, $[\mathcal{O} \omega]_{\alpha_{\mathscr{O}}(\omega)} \in C B(\mathscr{M})$.

Let $\omega_{1}, \omega_{2} \in \mathscr{M}$, then there exists $\alpha_{\mathscr{O}}\left(\omega_{1}\right), \alpha_{\mathscr{O}}\left(\omega_{1}\right] \in(0,1]$ such that $\left[\mathcal{O} \omega_{1}\right]_{\alpha_{\mathscr{O}}\left(\omega_{1}\right)},\left[\mathcal{O} \omega_{2}\right]_{\alpha_{\mathscr{O}}\left(\omega_{2}\right)}$ $\in C B(\mathscr{M})$. Let $\omega_{1} \in\left[\mathcal{O} \omega_{1}\right]_{\alpha_{\mathscr{O}}\left(\omega_{1}\right)}$ be arbitrary such that

$$
\varpi_{1}(\kappa) \in \mathfrak{h}(\kappa)+\int_{0}^{\kappa} \mathfrak{\Im}\left(\kappa, \tau, \omega_{1}(\tau)\right) \mathrm{d} \tau,
$$

for $\kappa \in[0,1]$ holds. This means that for all $\kappa, \tau \in[0,1]$, there exists $\dot{\mathfrak{I}}_{\omega_{1}}(\kappa, \tau) \in \mathfrak{J}_{\omega_{1}}(\kappa, \tau)=\mathfrak{J}\left(\kappa, \tau, \omega_{1}(\tau)\right)$ such that

$$
\varpi_{1}(\kappa)=\mathfrak{h}(\kappa)+\int_{0}^{\kappa} \dot{\mathfrak{t}}_{\omega_{1}}(\kappa, \tau) \mathrm{d} \tau,
$$

for $\kappa \in[0,1]$. For all $\omega_{1}, \omega_{2} \in \mathscr{M}$, it follows from (b) that

$$
\left|\mathfrak{J}\left(\kappa, \tau, \omega_{1}\right)-\mathfrak{J}\left(\kappa, \tau, \omega_{2}\right)\right|^{p} \leq l(\tau)\left|\omega_{1}(\tau)-\omega_{2}(\tau)\right| .
$$

It means that there exists $z(\kappa, \tau) \in \mathfrak{J}_{\omega_{2}}(\kappa, \tau)$ such that

$$
\left|\dot{\mathrm{j}}_{\omega_{1}}(\kappa, \tau)-z(\kappa, \tau)\right|^{p} \leq l(\tau)\left|\omega_{1}(\tau)-\omega_{2}(\tau)\right|,
$$

for all $\kappa, \tau \in[0,1]$.

Now, we can take the set-valued operator $U$ defined by

$$
U(\kappa, \tau)=\widetilde{\mathfrak{J}}_{\omega_{2}}(\kappa, \tau) \cap\left\{u \in \mathbb{R}:\left|\dot{\mathfrak{j}}_{\omega_{1}}(\kappa, \tau)-u\right| \leq l(\tau)\left|\omega_{1}(\tau)-\omega_{2}(\tau)\right|\right\}
$$

Hence, by (a), $U$ is lower semicontinuous. It follows that there exists a continuous operator $\dot{\mathrm{I}}_{\omega_{2}}(\kappa, \tau):[0,1] \times[0,1] \longrightarrow \mathbb{R}$ such that $\dot{\mathfrak{I}}_{\omega_{2}}(\kappa, \tau) \in U(\kappa, \tau)$ for $\kappa, \tau \in[0,1]$. Then, $\Phi_{2}(\kappa)=\mathfrak{h}(\kappa)+\int_{0}^{\kappa} \dot{\mathfrak{l}}_{\omega_{1}}(\kappa, \tau) \mathrm{d} \tau$ satisfies that

$$
\varpi_{2}(\kappa) \in \mathfrak{h}(\kappa)+\int_{0}^{\kappa} \mathfrak{J}\left(\kappa, \tau, \omega_{2}(\tau)\right) \mathrm{d} \tau, \quad \kappa \in[0,1]
$$

That is $\Phi_{2} \in\left[\mathcal{O} \omega_{2}\right]_{\alpha_{\mathscr{O}}\left(\omega_{2}\right)}$ and

$$
\begin{aligned}
& \left|\varpi_{1}(\kappa)-\omega_{2}(\kappa)\right|^{p} \leq\left(\int_{0}^{\kappa}\left|\dot{\mathrm{t}}_{\omega_{1}}(\kappa, \tau)-\dot{\mathrm{i}}_{\omega_{2}}(\kappa, \tau)\right| \mathrm{d} \tau\right)^{p} \\
& \quad \leq\left(\int_{0}^{\kappa} l(\tau)\left|\omega_{1}(\tau)-\omega_{2}(\tau)\right| \mathrm{d} \tau\right)^{p} \leq \max _{\tau \in[0,1]}^{p}|\omega(\tau)-\omega(\tau)|^{p}\left(\int_{0}^{\kappa} l(\tau) \mathrm{d} \tau\right) \leq \frac{k^{2}}{2^{p-1}} d_{b}\left(\omega_{1}, \omega_{2}\right),
\end{aligned}
$$

for all $\kappa, \tau \in[0,1]$. Thus, we obtain that

$$
2^{p-1} d_{b}\left(\omega_{1}, \omega_{2}\right) \leq k^{2} d_{b}\left(\omega_{1}, \omega_{2}\right) .
$$

Interchanging the roles of $\omega_{1}$ and $\omega_{2}$, we obtain that

$$
s H_{b}\left(\left[\mathcal{O} \omega_{1}\right]_{\alpha_{\mathcal{O}}\left(\omega_{1}\right)},\left[\mathcal{O} \omega_{2}\right]_{\alpha_{\mathcal{O}}\left(\omega_{2}\right)}\right) \leq k^{2} d_{b}\left(\omega_{1}, \omega_{2}\right) \text {. }
$$

This implies that

$$
\sqrt{s H_{b}\left(\left[\mathcal{O} \omega_{1}\right]_{\alpha_{\mathscr{O}}\left(\omega_{1}\right)},\left[\mathcal{O} \omega_{2}\right]_{\alpha_{\mathscr{O}}\left(\omega_{2}\right)}\right)} \leq k \sqrt{d_{b}\left(\omega_{1}, \omega_{2}\right)} .
$$

Taking exponential, we have

$$
e^{\sqrt{s H_{b}\left(\left[\mathcal{O} \omega_{1}\right]_{\alpha_{\mathcal{O}}\left(\omega_{1}\right)},\left[\mathcal{O} \omega_{2}\right]_{\alpha_{\mathcal{O}}\left(\omega_{2}\right)}\right)}} \leq e^{k \sqrt{d_{b}\left(\omega_{1}, \omega_{2}\right)}} .
$$

Taking the function $\Theta \in \Omega_{s}$ defined by $\Theta(\eta)=e^{\sqrt{\eta}}$ for $\eta>0$, we get that the condition (8) is satisfied. Using the result 6 , we conclude that (54) has a solution.

\section{Data Availability}

The data used to support the findings of this study are available from the corresponding author upon request.

\section{Conflicts of Interest}

The authors declare that they have no conflicts of interest.

\section{References}

[1] S. Czerwik, "Contraction mappings in b-metric spaces," Acta Mathematica et Informatica Universitatis Ostraviensis, vol. 1, pp. 5-11, 1993.

[2] S. Czerwik, K. Dlutek, and S. L. Singh, "Round-off stability of iteration procedures for operators in b-metric spaces," Nature Physics, vol. 11, pp. 87-94, 1997.

[3] S. Czerwik, "Nonlinear set-valued contraction mappings in b-metric spaces," Atti del Seminario Matematico e Fisico dell' Universita di Modena e Reggio, vol. 46, pp. 263-276, 1998.

[4] M. Cosentino, M. Jleli, B. Samet, and C. Vetro, "Solvability of integrodifferential problems via fixed point theory in b-metric spaces," Fixed Point Theory Apply, vol. 2015, p. 70, 2015.

[5] J. R. Roshan, V. Parvaneh, and Z. Kadelburg, "Common fixed point theorems for weakly isotone increasing mappings in ordered b-metric spaces," Journal of Nonlinear Sciences and Applications, vol. 07, no. 04, pp. 229-245, 2014.

[6] H. Qawaqneh, M. Md Noorani, W. Shatanawi, H. Aydi, and H. Alsamir, "Fixed point results for multi-valued contractions in b-Metric spaces and an application," Mathematics, vol. 7, no. 2, p. 132, 2019. 
[7] E. Ameer, H. Aydi, M. Arshad, H. Alsamir, and M. Noorani, "Hybrid multivalued type contraction mappings in $\alpha \mathrm{k}$ complete partial b-metric spaces and applications," Symmetry, vol. 11, no. 1, p. 86, 2019.

[8] M. Jleli and B. Samet, "A new generalization of the Banach contraction principle," Journal of Inequalities and Applications, vol. 38, pp. 1-8, 2014.

[9] H. A. Hançer, G. Minak, G. Minak, and I. Altun, "On a broad category of multivalued weakly Picard operators," Fixed Point Theory, vol. 18, no. 1, pp. 229-236, 2017.

[10] B. A. S. Alamri, R. Agarwal, and J. Ahmad, "Some new fixed point theorems in b-metric spaces with application," Mathematics, vol. 8, no. 5, p. 725, 2020.

[11] W. Kumam, P. Sukprasert, P. Kumam, A. Shoaib, A. Shahzad, and Q. MAhmood, "Some fuzzy fixed point results for fuzzy mappings in complete b-metric spaces," Cogent Mathematics \& Statistics, vol. 5, pp. 1-12, 2018.

[12] J. Ahmad, A. E. Al-Mazrooei, and I. Al-Mazrooei, "Generalized $\Theta$-contractive fuzzy mappings," Journal of Intelligent \& Fuzzy Systems, vol. 35, no. 2, pp. 1935-1942, 2018.

[13] H. H. Al-Sulami, J. Ahmad, N. Hussain, and A. Latif, "Solutions to fredholm integral inclusions via generalized fuzzy contractions," Mathematics, vol. 7, no. 9, p. 808, 2019.

[14] S. Heilpern, "Fuzzy mappings and fixed point theorem," Journal of Mathematical Analysis and Applications, vol. 83, no. 2, pp. 566-569, 1981.

[15] J. Ahmad, A. Azam, and S. Romaguera, "On locally contractive fuzzy set-valued mappings," Journal of Inequalities and Applications, vol. 2014, no. 1, p. 74, 2014.

[16] A. E. Al-Mazrooei and J. Ahmad, "Fixed point theorems for fuzzy mappings with applications," Journal of Intelligent \& Fuzzy Systems, vol. 36, no. 4, pp. 3903-3909, 2019.

[17] A. A. N. Abdou and J. Ahmad, "Multivalued fixed point theorems for $\mathrm{F}_{-}\{\backslash \mathrm{rho}\} \$$-contractions with applications to volterra integral inclusion," IEEE Access, vol. 7, pp. 146221146227, 2019.

[18] P. Borisut, P. Kumam, V. Gupta, and N. Mani, "Generalized $(\psi, \alpha, \beta)$-Weak contractions for initial value problems," Mathematics, vol. 7, no. 3, p. 266, 2019.

[19] V. Gupta, W. Shatanawi, and N. Mani, "Fixed point theorems for $\$ \$(\backslash p s i$, lbeta $) \$ \$(\psi, \beta)$-Geraghty contraction type maps in ordered metric spaces and some applications to integral and ordinary differential equations," Journal of Fixed Point Theory and Applications, vol. 19, no. 2, pp. 1251-1267, 2017.

[20] P. Patle, D. Patel, H. Aydi, and S. Radenović, "ON H+Type multivalued contractions and applications in symmetric and probabilistic spaces," Mathematics, vol. 7, no. 2, p. 144, 2019. 